\title{
La comunicación como unidad de análisis en Luhmann y Habermas
}

\author{
Communication as analytical unit in Luhmann and Habermas \\ Sergio Pignuoli-Ocampo / spignuoli@conicet.gov.ar \\ Consejo Nacional de Investigaciones Cientificas y Técnicas y \\ Universidad de Buenos Aires, Argentina \\ (CONICET-UBA-IIGG)
}

\begin{abstract}
The present paper compares the commuicative definitions of "the social" proposed by Niklas Luhmann and Jürgen Habermas from a systematic perspective. From the results reached, the reciprocal receptions between the authors are discussed and a renewed theoretical interpretation of divergences and convergences is proposed. The hypothesis of the work states that Habermas and Luhmann, based in the concept of communication, converge in a radically dyadic and multi-leveled conception of the sociological analytical unit, however they diverge in its analytical segmentation.
\end{abstract}

Key words: Luhmann, Habermas, communication, dyadism.

Resumen: Este trabajo compara desde una perspectiva sistemática las definiciones comunicativas de "lo social" propuestas por Niklas Luhmann y Jürgen Habermas. A partir de los resultados alcanzados se discuten las recepciones recíprocas de los autores y se propone una renovación de la interpretación teórica de divergencias y convergencias. La hipótesis es que Habermas y Luhmann convergen en una concepción radicalmente diádica y multinivelada de la unidad de análisis sociológica sobre la base del concepto de comunicación, pero divergen en la segmentación analítica que hacen de ella.

Palabras clave: Luhmann, Habermas, comunicación, diadismo. 


\section{Introducción: la comunicación como unidad de análisis de la sociología}

En este trabajo cotejamos las definiciones de "lo social" de la Teoría General de Sistemas Sociales (TGSS) de Niklas Luhmann y de la Teoría de la Acción Comunicativa (TAC) de Jürgen Habermas. Nos interesa abordar en particular la redefinición comunicativa de "lo social" realizada por ambos autores entre los años setenta y ochenta. En ellas se observan varios puntos y aspectos en común. Nuestro objetivo es identificarlos, relevarlos y compararlos, a fin de extraer de ellos consecuencias teóricas fundamentales relativas a la unidad de análisis de la sociología.

Esos puntos en común quedan, sin embargo, solapados habitualmente detrás de la autodenominada confrontación entre "paradigmas", que prima en los diálogos entre la TGSS y la TAC. De manera que, para comparar aquellas redefiniciones, asumimos la necesidad de una perspectiva de análisis que trascienda esa petrificada polémica y sea capaz de establecer un esquema de comparación integral y de focalizar y desagregar definiciones complejas de "lo social". Para ello en este trabajo asumimos una perspectiva sistemática focalizada en el cotejo de unidades de análisis.

El debate Luhmann-Habermas es uno de los más importantes de la sociología europea de posguerra. Su riqueza conceptual es notable, ya sea porque fue abonado por los mismos autores durante tres décadas, o por la amplitud de los ejes de la controversia que se sucedieron a través de los años (intersubjetividad, racionalidad, funcionalismo, dialogismo, normatividad, etc.), o por la vasta bibliografía acumulada desde la década de 1970 (sólo a título ilustrativo: Maciejewski, 1974; Söllner, 1982; Rasch, 2000; Bonacker, 2002; Bolz, 2012).

Al evaluarlo, sin embargo, desde nuestro objetivo comparativo, el debate no ofrece contribuciones sistemáticas. En él primaron, y priman aún, las recepciones recíprocas, es decir, trabajos exclusivamente interesados en fortalecer una posición del debate y rebatir a la otra, pero sin propósitos sistemáticos, desinteresados de las comparaciones integrales de sendos programas de investigación. Por esta razón no extraña que, dentro de la historia del debate, los esfuerzos sistemáticos sean relativamente menores en volumen y recientes en el tiempo.

Estos trabajos corrieron del centro de atención a las recepciones recíprocas para concentrarse en las convergencias de la TGSS y la TAC. Operación de la cual surgieron resultados tan destacados como promisorios. Podemos mencionar, entre otros, los trabajos de Strydom (1999), Chernilo (2002) y Mascareño (2009). Strydom identificó un espectro delimitado de conver- 
gencias entre ambos programas en la recepción de Parsons, a partir del cual planteó la triple contingencia. Chernilo diseñó un esquema general de comparación para los medios de comunicación simbólicamente generalizados, con él cotejó las propuestas de Luhmann y Habermas y halló divergencias y convergencias. Con resultados análogos, Mascareño amplió la propuesta de Chernilo con el problema de la emergencia y, además, extendió el corpus de autores tratados.

Nuestro proyecto de investigación se inscribe en esta línea. Nos proponemos analizar con un propósito sistemático los principales programas de la sociología europea de la posguerra tardía y uno de los focos cae sobre la TAC y la TGSS, debido a la profundidad con que redefinieron el sistema de categorías de la sociología a partir de la comunicación y a la pretensión de generar un "cambio de paradigma" en la disciplina a partir de ello. En ese marco comparamos de manera integral los fundamentos teóricos generales (ontológicos, epistemológicos, metodológicos, sociológicos, discursivos) de ambos programas.

En este trabajo recogemos algunos resultados ya alcanzados. Sabemos, en relación con el componente ontológico, que los dos programas, junto con el de Latour, convergen en el rechazo del por ellos denominado "proyecto ontológico de Occidente" y en la aceptación de una estructuración de lo real ontológicamente débil y fácticamente fuerte (Pignuoli-Ocampo, 2016b). Ya en el terreno de la sociología, partimos de la base, un tanto genérica y requerida de precisión y detalle, de que Habermas y Luhmann rechazan de manera convergente la reducción tanto colectivista como accionalista del concepto de comunicación (Pignuoli-Ocampo y Zitello, 2008).

Asimismo, contamos con la hipótesis avanzada de que ambos convergen en ubicar la relación entre estructuras de sentido y unidades de análisis en un nivel de estructura específico, donde las segundas guardan con las primeras una relación de presunción condicional, de modo que las propiedades de sentido de la estructura carecen de poder causal sobre las unidades, como se observa en los conceptos de intersubjetividad de Habermas y de intepenetración de Luhmann (Paladino y Pignuoli-Ocampo, 2014). Por último, encuadramos estos antecedentes dentro de la tesis de que los componentes performativos con pretensión de cambio paradigmático de la TGSS y la TAC son similares.

Estos componentes se dirigieron contra la "crisis de la sociología", a los efectos de robustecer su intervención en la competencia de diagnósticos y alternativas del contexto disciplinario, y así invistieron sus innovaciones conceptuales con el estatuto y las pretensiones de un "cambio de paradigma" (Pignuoli-Ocampo, 2015b). 
Llegó el momento de mudar el foco comparativo a uno de los problemas fundamentales de la sociología: la definición de unidad de análisis, es decir, las elaboraciones conceptuales dedicadas a responder la pregunta: “¿Qué es lo social?" Entendemos por definición de "lo social" una elaboración conceptual que supone la estructuración de lo real e introduce en ella la diferenciación cualitativa de un orden de realidad específico al que indica autológicamente como "social". Toda definición de "lo social" delimita así el campo de fenómenos y el rango de problemas que considerara como objeto de la sociología.

A causa del predominio de las recepciones recíprocas, la determinación del método para comparar dichas definiciones forma parte del problema de investigación. En este trabajo asumimos la perspectiva multinivelada del programa de investigación como metodología sistemática, que ya empleé para cotejar los fundamentos sociológicos de Luhmann y Latour (Pignuoli-Ocampo, 2016a) y de Husserl, Schutz y Luhmann (Pignuoli-Ocampo, 2015a). ${ }^{1}$ Este diseño sistemático, en línea con Ritzer (2001) y Mascareño (2008), compara arquitecturas teóricas.

Se asume que las teorías sociológicas conceptualizan un núcleo común de problemas fundamentales hacia los cuales la elaboración de conceptos se orienta y sobre los que organizan su arquitectura teórica. Así vistos, los conceptos que se orientan hacia un mismo problema disciplinario son equivalentes funcionales y resultan comparables, aunque se inscriban en materiales diversos y empleen terminologías heterogéneas. Desde esta perspectiva podemos establecer equivalencias funcionales entre las definiciones de la TAC y TGSS y cotejarlas, evitando los equívocos y falacias homonímicas de los cotejos puramente terminológicos.

Como es sabido, desde mediados de los años setenta y sobre todo desde los ochenta, tanto Habermas como Luhmann relanzaron sus programas a partir de un conjunto de innovaciones conceptuales basadas en la redefinición comunicativa de "lo social", es decir, a partir de establecer teóricamente a la comunicación como unidad de análisis general de la sociología.

En el caso de Luhmann, dicha definición se centra en el modelo sintético de la comunicación. Este supone un criterio de socialidad fundado en el mutualismo, un planteo condicional basado en la doble contingencia y una unidad de análisis segmentada en el concepto de comunicación como síntesis de tres selecciones. En el caso de Habermas, esta definición se centra en el modelo de generación de entendimiento de la acción comunicativa. Este supone un criterio de socialidad fundado en el reconocimiento intersubjetivo, un plan-

1 Para la fundamentación epistemológica y metodológica de la perspectiva remito a Pignuoli-Ocampo (2017). 
teo condicional basado en la fuerza ilocutiva del lenguaje y una unidad de análisis segmentada en el concepto de acción comunicativa como éxito ilocutivo.

Dada la complejidad de estas definiciones comunicativas de "lo social", nuestra propuesta metodológica consiste en desagregarlas en tres aspectos básicos: criterio de socialidad, planteo condicional y unidad de análisis. El primero es el criterio de socialidad que remite a las propiedades distintivas, necesarias y suficientes, para predicar, definir y delimitar la imputación de la cualidad sociológica a una construcción referencial determinada: la TGSS lo elaboró en torno al concepto de mutualismo y la TAC, en torno al reconocimiento intersubjetivo.

El segundo remite a las condiciones de posibilidad que, a juicio de los autores, son necesarias pero no suficientes para la formación de entidades sociales dentro del mundo: la TGSS lo elaboró en torno al concepto de doble contingencia y la TAC, en torno al concepto de fuerza ilocutiva del lenguaje. El tercero es la determinación de la unidad de análisis, que segmenta una unidad, la relaciona con sus condiciones y describe sus propiedades sociológicas como entidad social dentro del mundo: para la TGSS esa unidad es el concepto de comunicación como síntesis de tres selecciones y para la TAC, la acción comunicativa como éxito ilocutivo. En la Tabla $1^{2}$ sintetizamos las equivalencias.

Nuestra hipótesis es que los autores convergen en materia de criterios de socialidad, planteos condicionales y de unidad de análisis, debido a la común asunción de un esquema sociológico marcadamente diádico en todos los tres niveles, mientras que divergen en gran medida en torno a la segmentación de dichas unidades de análisis.

La selección de materiales no trajo dificultades. Seleccionamos materiales de Luhmann pertenecientes al "periodo autopoiético", iniciado en los años ochenta, en los que formuló las categorías relativas a la comunicación, la emergencia de sistemas sociales y la autopoiesis. Este corpus se centró en la principal obra del periodo: Soziale Systeme (Luhmann, 1984) y lo ampliamos con un artículo posterior que lo refuerza (Luhmann, 1990).

En cuanto a Habermas, seleccionamos materiales del "giro lingüístico", que comenzó en los años setenta y alcanzó su punto culminante en Theorie des kommunikativen Handelns (Habermas, 1981), donde construyó un objeto sociológico basado en la relación entre acción comunicativa y racionalidad orientada al entendimiento. Ampliamos con trabajos preparatorios (Habermas, 1984) y posteriores (Habermas, 2002).

2 Las tablas 1 y 2 se encuentran en el Anexo, al final del presente artículo (Nota del editor). 
La exposición sigue este plan: ampliamos la discusión de la tensión entre recepciones recíprocas y abstracción requerida para realizar un análisis sistemático de unidades de análisis, luego cotejamos cada eje por separado: criterios de socialidad, planteos condicionales y unidades de análisis. Finalmente extraemos las conclusiones.

\section{Discusión preliminar: recepciones recíprocas y abstracción sistemática requerida}

Nuestro objetivo comparativo enfrenta un obstáculo importante: dialogar críticamente con el primado de las recepciones recíprocas entre los autores. Este obstáculo se observa en lo que Colomy (1991) denominó estrategias de imputación entre teorías contemporáneas. Los autores y programas de investigación mantienen diálogos y recepciones coincidentes o disidentes con otros autores y programas contemporáneos. Estas críticas e imputaciones realizadas recíprocamente entre los autores se convierten en un obstáculo cuando la comparación se circunscribe a ellas, en detrimento de una exploración amplia y sistemática de convergencias y divergencias entre ellos. Identificar este tipo de dificultad es especialmente relevante para plantear una comparación teórica entre Luhmann y Habermas, que sea capaz de dialogar críticamente con las recepciones que los autores mismos se prodigaron.

Como es sabido, las polémicas entre Jürgen Habermas y Niklas Luhmann son uno de los intercambios intelectuales más importantes en el campo de las ciencias sociales de la segunda mitad del siglo XX. Su riqueza teórica es innegable. Sin embargo, un tratamiento comparativo sistemático de sus propuestas no puede reducirse al comentario de dichas recepciones, pues ellas no sólo son ricas en aportes, sino también en omisiones, incorrecciones y elisiones. Un ejemplo es la elisión que ambos se prodigaron al respecto de la interacción.

Para Habermas (1981-II: 557; 1984: 24), Luhmann fue un sistémico holista que radicalizó la racionalidad funcionalista. Para Luhmann (1990: 6), Habermas fue un accionalista de la comunicación, renovador del racionalismo emancipatorio. Habermas elidió el esfuerzo de Luhmann por construir un emergentismo diádico de la comunicación, y éste, inversamente, elidió el esfuerzo de aquél por estudiar las estructuras generales del entendimiento sobre la base de la articulación (y no de la identificación) entre actos de habla y acción. 
Una corrección sistemática de esta recepción debe reponer los elementos elididos e indagar divergencias y convergencias por igual. En este caso se observa que ambos programas, sin desmedro de sus divergencias, convergen tanto en el rechazo de las tradiciones individualista y colectivista, como en la defensa de la antecedencia del interaccionismo sobre la tercera posición radical. En vista de ello revisaremos, por un lado, las divergencias con el objetivo de precisar aquellos aspectos en que, efectivamente, haya posiciones enfrentadas; y, por otro, aquellos aspectos menos enfrentados, con mayores coincidencias, que constituyen núcleos teóricos más abiertos a convergencias conceptuales.

Nuestra propuesta requiere un esquema comparativo con abstracción suficiente para identificar equivalencias funcionales entre terminologías heterogéneas relativas a la unidad de análisis de sociología. Para ello retomaremos el esquema que ya empleamos para cotejar los fundamentos sociológicos de Latour y Luhmann (Pignuoli-Ocampo, 2016a). Partimos del reciente debate sobre las dimensiones de las concepciones de socialidad (Heintz, 2004; Lindemann, 2006; Bedorf et al., 2010; Albert et al., 2010, entre otros). El supuesto del debate es que la sociología atraviesa una etapa multiparadigmática, donde conviven distintas definiciones del objeto disciplinario y se discute la posibilidad de compararlas desde una perspectiva metateórica.

Para ello se propone el concepto de dimensión de socialidad sobre el cual fundan un esquema comparativo. Se considera dimensión de socialidad a la unidad de magnitud con que es definida teóricamente la cualidad social en el marco de una concepción del objeto sociológico y se asume que las dimensiones fundamentales son tres: 1) una dimensión monádica, cuya unidad son las propiedades sociales atribuidas a los individuos y/o a sus acciones y/o a sus representaciones; 2) una dimensión diádica, cuya unidad son las propiedades sociales atribuidas a la constelación ego / alter ego; y 3) una dimensión triádica, cuya unidad son las propiedades sociales atribuidas a un término considerado tercero (tertium) respecto de los individuos, anterior y externo a ellos, capaz de organizarlos.

Nuestra propuesta consiste en, primero, ubicar las definiciones en el espacio de propiedades conformado por estas tres dimensiones de la socialidad. Segundo, desagregarlas analíticamente de acuerdo con la/s unidad/es de magnitud que la/s rija. Tercero, interpretarlas y compararlas según su distribución en las dimensiones y al juego que establezcan entre ellas. El modelo de análisis básico considera monadismo a las concepciones donde primen las definiciones monádicas, diadismo a aquellas donde preponderen las definiciones diádicas y triadismo cuando primen las definiciones triádicas. Acepta asimismo combinaciones entre ellas. 


\section{Criterios de socialidad}

En esta sección cotejamos los criterios de socialidad de la TGSS y la TAC. Los presentaremos por separado: el mutualismo y el reconocimiento intersubjetivo. Luego deslindaremos convergencias y divergencias.

\section{TGSS: mutualismo}

El mutualismo es el criterio específico de la emergencia por constitución múltiple de "lo social". Mutualismo significa que la alteración (Änderung) de los estados de uno corresponde con la alteración de los estados del otro (Luhmann, 1984: 65-66). Ello requiere procesadores autorreferenciales que procesan sentido y comprenden (verstehen) y la formación de una situación específica a partir del concurso de ellos, dentro de la cual son capaces de co-orientarse.

Por esta razón Luhmann establece el mutualismo como premisa general de la constitución múltiple (constitución mutualista o dialógica) de "lo social" y como criterio de socialidad de la TGSS. En cuanto tal tiene propiedades emergentes: cumple con las legalidades del mundo (termodinámicas, físicas, químicas, bioquímicas, orgánicas y psíquicas), pero implica la aparición cualitativamente novedosa de la co-orientación mutualista.

$\mathrm{Al}$ estar fundado en una constelación alter ego / alter ego de los procesadores autorreferenciales, el mutualismo tiene propiedades diádicas. No puede ser satisfecho por la unilateralidad de un sistema, pues requiere el concurso de, al menos, otro alter ego, no es, por ende, un criterio monádico ni requiere el concurso de un tercero (tertium) que genere la constitución múltiple y ordene la constelación; por tanto, ésta tampoco sigue un criterio triádico, ya que se encuentra regida por los principios de autorreferencia y tercero excluido.

\section{TAC: reconocimiento intersubjetivo (intersubjektive Anerkennung)}

El reconocimiento intersubjetivo es el acto de reconocimiento del oyente como un alter ego por parte del hablante, el que es realizado mediante la expresión de un acto de habla ilocutivo. Al reconocer intersubjetivamente a otro sujeto es reconocida de suyo, primero, su capacidad de acción como premisa para la coordinación cooperativa; segundo, su competencia para comprender o inteligir proposiciones y pretensiones; y tercero, su capacidad 
para aceptar o rechazar y criticar la validez de la pretensión ofertada. Habermas subrayó que la ilocución tiene un "efecto vínculo" (Bindungseffekt) entre actores que se da con el fin de entenderse para coordinar sus acciones y destacó que la realización del reconocimiento intersubjetivo es la propiedad sociológica fundamental del componente ilocutivo, debido a que fija la naturaleza social de su realización. En cuanto tal tiene propiedades generativas, pues pone-en-acto al reconocimiento dentro del mundo mediante un acto de habla.

Esta actualización ilocutiva performa la situación de habla en el mundo con la forma de una constelación alter ego / alter ego entre los sujetos recíprocamente reconocidos gracias a ella y genera un horizonte de entendimiento entre ambos, por esta razón el reconocimiento intersubjetivo tiene propiedades diádicas. Éste no puede ser alcanzado por los sujetos aislados, pues ni las fuerzas ni los actos ilocutivos son creados ex nibilo por ningún sujeto ni están alojados en él o en su naturaleza interior, sino que están alojados en el lenguaje natural, cuya teleología se encuentra orientada al entendimiento.

El reconocimiento, por ende, no depende de un individuo aislado, él sólo desarrolla la competencia del lenguaje para emplearlo, por tanto, no es monádico. Asimismo, la puesta en acto ilocutiva supone las fuerzas ilocucionarias como potencia dada, pero ella no es determinada ni causada por tal potencia. Así, el paso de la potencia al acto de los sujetos en situación de habla no es causado ni determinado por el lenguaje, sino por la propia situación generada por la ejecución de actos ilocutivos en un momento y un lugar determinados. El lenguaje es el Medium, no interviene de manera causal en la constitución de la situación ni es su razón eficiente, por ello no posee estatuto triádico, ni, por ende, requiere un criterio de socialidad con tal estatuto.

\section{Convergencias}

Hallamos dos convergencias significativas: 1) la base interaccional, situacional y recíproca y 2) la centralidad de la alteridad. La primera trata de la convergencia de ambos programas en torno a la base única, interaccional, situacional y recíproca del criterio de socialidad. En cuanto al carácter interaccional, ellos rechazan los criterios de base individual o supraindividual y abrazan una base interaccional, debido a que exigen el concurso de, al menos, dos agentes, como se observa en las definiciones de mutualismo de la TGSS y reconocimiento intersubjetivo de la TAC.

Esta exigencia no puede ser satisfecha al nivel individual porque el concurso no puede reducirse a uno de ellos en singular, tampoco puede satisfacerse al nivel supraindividual porque la base no es independiente de 
dicho concurso. El concurso es la base interaccional que establece una base única (ni dual ni fragmentada) de composición múltiple para el criterio de socialidad. Esta convergencia es de primera importancia porque establece la no-contradicción entre los programas en cuanto a la definición de "lo social".

En cuanto al carácter situacional, los programas anclan temporal y espacialmente el concurso: éste sucede dentro del mundo, en un punto del tiempo y con una temporalidad determinados, en un segmento de extensión y una espacialidad determinados. Ese tiempo y espacio dotan al concurso de unidad situacional. Respecto a la reciprocidad de perspectivas, Luhmann y Habermas convergen en que la unidad situacional del concurso no es mera co-existencia objetiva de agentes, sino formación de perspectivas recíprocas en los concursantes. Ello implica dislocación de perspectivas egocéntricas y apertura de perspectivas orientadas al otro agente y viceversa. Esta reciprocidad no supone conformidad ni mucho menos armonía entre las perspectivas recíprocas, sino únicamente atención mutua.

La segunda convergencia es que Luhmann y Habermas incluyen un componente de alteridad en un lugar central de sus planteos. Para ellos la concepción sociológica de la alteridad no sólo debe considerar la construcción de alter desde la perspectiva de ego, sino también y más fundamentalmente la construcción de ego como alter ego de su alter ego dentro de la situación de interacción y viceversa. Esta duplicidad es irreductible y sigue su propia dinámica en ese marco. Esta dislocación radical de las perspectivas egocéntricas es el componente de alteridad que ambos programas incorporan en sus criterios de socialidad.

\section{Divergencia}

En este elemento hallamos una divergencia significativa entre los acentos interaccionistas de los planteos. Ambos consideran la interacción como base del criterio de socialidad, pero los acentos sobre los mismos componentes difieren. Habermas la considera primordial para la acción comunicativa, es su plataforma y su unidad. Para Luhmann es una de las posibilidades del mutualismo, no es la única y en cuanto doble contingente puede ser desacoplada espacio-temporalmente, sin perder su unidad. 


\section{Planteos condicionales}

En esta sección cotejamos los planteos condicionales de la TGSS y la TAC. Los presentaremos por separado: doble contingencia y fuerzas ilocucionarias e identidad de significados. Luego deslindaremos convergencias y divergencias.

\section{TGSS: doble contingencia}

La doble contingencia es la "transición" hacia los sistemas sociales (Luhmann, 1984: 148). Ella es condición necesaria, pero no suficiente, de la emergencia de sistemas sociales, puesto que posee la propiedad mutualista, pero no es comunicativa ni posee unidad operativa. En función del problema de cómo es posible el orden social, la categoría de doble contingencia indica la emergencia de un umbral de irreductibilidad del sentido social.

La categoría establece tres condiciones para la formación de ese umbral, que ofician de dimensiones de la categoría, a saber: 1) un problema específico (la indeterminación del comportamiento del otro); 2) una experiencia plena de sentido (la co-experiencia de la recíproca alteridad); y 3) la emergencia de un orden de realidad situacionalmente diferenciado.

En cuanto al problema, hay doble contingencia si, y sólo si, se conforma el problema de la indeterminación del sentido social de la acción. Éste surge cuando al menos dos sistemas se comprenden y asumen el comportamiento del otro sistema como premisa de su propio comportamiento. En ese momento, surgen simultáneamente la imposibilidad de determinar con grado de certeza el comportamiento del otro, y, correlativamente, la indeterminación del propio comportamiento.

Este es un problema concreto y constante (Dauerproblem) desde la perspectiva de los alter ego (o actores), quienes deben lidiar con él de un modo autorreferencial. Al tratarlo producen efectos incapaces de resolverlo definitivamente, pero que establecen una referencia de sentido, que posibilita que en otro punto del tiempo el problema pueda volver a ser tratado, desde el estado generado por ese efecto.

En cuanto experiencia, la doble contingencia supone la co-experiencia de la irreductible diferencia y alteridad del otro. Esta experiencia implica que los sistemas participantes co-experimentan la divergencia entre sus perspectivas. Esto provoca un círculo de expectativas reflexivas que los fuerza a seleccionar con arreglo a ellas. En cuanto emergente, la doble contingencia implica la emergencia de la dimensión social del sentido y la emergencia de una zona de selección. La primera forma el doble horizonte ego/alter y disloca las expecta- 
tivas ego-céntricas. La segunda forma un horizonte situacional que coacciona a la selección, y en cuanto tal es irreductible a los sistemas participantes.

Cuando se cumplen las tres condiciones emerge una forma de sentido y un modo de contingencia específica e irreductiblemente sociales dentro del mundo. Ellos organizan la doble contingencia con la forma de expectativa reflexiva: "Yo hago lo que tú quieres, si tú haces lo que yo quiero", cuya forma negativa, en caso de conflicto, es: "Yo no hago lo que quieres, porque tú no haces lo que yo quiero". Esta definición sociológica es lo que Luhmann (1984: 153) denominó teorema de la doble contingencia, y por cuanto establece una forma situacional de forzamiento mutualista a seleccionar plantea la condición de sentido necesaria de la comunicación y se conserva como condición permanente de ella. ${ }^{3}$

\section{TAC: fuerzas ilocucionarias e identidad de significados}

El planteo condicional de la TAC reposa sobre la relación interna entre intersubjetividad y lenguaje. Éste condiciona la intersubjetividad doblemente. En primer lugar, se aloja en la relación del reconocimiento intersubjetivo con las fuerzas y actos ilocutivos. ${ }^{4}$ En segundo lugar, es la sede de identidad de significados asociados con significados intersubjetivamente reconocidos como compartidos. Uno y otro condicionamiento implican sendas definiciones de intersubjetividad.

La primera definición asocia la generación de intersubjetividad con la teoría de los actos de habla, la segunda asocia el supuesto de intersubjetividad con la teoría del significado. Habermas las articula de manera sistemática en el concepto de reconocimiento intersubjetivo, el que, por ende, resulta complejizado.

En cuanto a la primera definición, Habermas (1981-I: 430) asume que las fuerzas ilocucionarias (illokutionärer Kräfte) son los puntos nodales de las redes de societalización (Vergesellschaftung) en la medida en que ofrecen esquemas para la elaboración (Herstellung) de relaciones interpersonales. Estas fuerzas constituyen la infraestructura societal del lenguaje y están ellas mismas en flujo. El léxico ilocutivo se encuentra alojado en el lenguaje natural, por

3 Para presentaciones más amplias del concepto de doble contingencia remito a Lindemann (1999), Vanderstraeten (2002), Pignuoli-Ocampo (2013b), entre otros.

4 La noción de fuerza ilocucionaria proviene de la pragmática de los actos de habla. En Austin (1982: 117), las fuerzas de una expresión designan los modos en que hay que entender una proposición, "cómo hay que tomarlas". Habermas reinterpretó sociológicamente el potencial de reconocimiento supuesto en ellas. 
tanto varía según cada lengua con formas gramaticales consolidadas o modos más flexibles como verbos performativos, partículas oracionales, entonación de frases

Habermas las sitúa en el planteo condicional debido a que las fuerzas ilocucionarias son condición necesaria pero no razón suficiente de los actos de habla ilocutivos. La distinción entre potencia y acto dentro del mismo Medium del lenguaje es clave: el lenguaje posibilita el efecto vínculo intersubjetivo al poner estas fuerzas a disposición en modo de potencial para los sujetos de habla e interacción, pero su puesta en acto depende de la ejecución (o no) de los ilocutivos por parte de los sujetos cuando se dan el fin intersubjetivo de coordinar sus planes de acción.

Respecto a la segunda definición, Habermas postula que el lenguaje es la sede de la semántica intersubjetiva, en cuanto aloja la identidad del significado de una comunidad de habla. ${ }^{5}$ La medida comunitaria de esta segunda definición de intersubjetividad fundamenta en un saber de fondo compartido, tendencialmente holista por ser inabarcable como totalidad, las pretensiones de validez de los actos de habla, pues pone en relación dichas pretensiones con una apertura y significación compartidas.

Tal es la base de validez del habla y Habermas la sitúa en el plano condicional, pues el reconocimiento intersubjetivo del significado compartido de las pretensiones de validez abre un horizonte de aceptabilidad, crítica y acuerdo para los actos de habla ejecutados por sujetos en situación de habla. De esta manera no fuerza el entendimiento entre ellos, sólo lo posibilita.

Habermas articula ambas definiciones de intersubjetividad en un punto específico de doble condicionamiento de la acción comunicativa: la generación ilocutiva de la situación de habla supone el reconocimiento intersubjetivo de pretensiones de validez con la forma de enunciados aceptables, bajo el supuesto contrafáctico de un entendimiento entre ellos.

\section{Convergencias}

En este elemento hallamos dos convergencias significativas: 1) el doble uso del criterio de socialidad y 2) la inclusión de un tercer término condicional. La primera convergencia es doble uso del criterio de socialidad de Luhmann y

5 Lafont (1993: 169) señaló que con la inclusión del mundo de la vida como saber de trasfondo, Habermas volvió de Humboldt a Gadamer y ya no consideró las condiciones de posibilidad del entendimiento sólo desde la perspectiva de los participantes en la comunicación (a fronte), sino también a partir de las condiciones de posibilidad que han de estar dadas (a tergo) para que éste pueda tener lugar. 
Habermas, quienes, por un lado, definieron con él la cualidad sociológica de la unidad de análisis, y, por otro, establecieron las condiciones de tales unidades. Es decir, para los programas las condiciones y las unidades cumplen el mismo criterio de socialidad, sin homologarse por ello, pues en ambos casos el planteo condicional fija las premisas fácticas y lógicas de las unidades y tiene una amplitud mayor.

La segunda convergencia se deriva de la anterior. Ambos programas plantean un tercer término condicional que posee cualidad social porque cumple el criterio de socialidad, pero no es una unidad social porque no es una unidad de análisis. Es más amplio que ellas, como es el caso de la doble contingencia en la TGSS y la fuerza ilocucionaria en la TAC. Al igual que las unidades, las condiciones son intramundanas.

Sin embargo, tienen una diferencia con aquéllas: las condiciones carecen de estatuto ontológico acontecimental, tienen un estatuto ontológico o medial (TAC) o problemático (TGSS), pero no son unidades. Sin condiciones sociales no habría unidades sociales, pero aquéllas no son unidades sociales. Así lo observamos en la doble contingencia: ella posibilita la comunicación, pero no es comunicación. Lo mismo vale para el reconocimiento intersubjetivo: él posibilita la acción comunicativa, sin ser una de tales acciones. Esta convergencia aclara la posición de los programas: las condiciones sociales permiten identificar el objeto sociológico, pero no equivalen al objeto.

\section{Divergencias}

La modalidad del planteo condicional es un problema sistemático muy interesante, pues es el punto de mayor divergencia entre las definiciones de lo social de nuestros programas. Los programas no difieren tanto en las bases diádicas ni en las unidades de análisis como lo hacen en las condiciones sociales. Observamos que Luhmann elabora un planteo condicional basado en el sentido social, caracteriza el tercer término (doble contingencia) como un problema fáctico, propone un esquema lógico basado en la transición y lo organiza en torno a la diferencia de las perspectivas incongruentes.

Habermas también basa su planteo en el sentido social, pero a diferencia de Luhmann caracteriza el tercer término (fuerza ilocucionaria) como una mediación, propone un esquema de potencia/acto para el lenguaje y lo organiza en torno a la identidad de significados. No es posible agrupar las posiciones: los autores coinciden en un aspecto, pero se contraponen en otro. Es interesante subrayar que las divergencias parten de un criterio de socialidad diádico centrado en la alteridad. Esto demuestra que, primero, las divergencias 
son muy marcadas, pero también lo son las convergencias y aquéllas parten de éstas; y segundo, que el diadismo ofrece una gran flexibilidad para la elaboración de la materia condicional.

\section{Unidades de análisis}

En esta sección cotejamos las definiciones de unidad de análisis de la TGSS y la TAC. Las presentaremos por separado: la comunicación entendida como "síntesis de tres selecciones" y la acción comunicativa entendida como "éxito ilocutivo". Luego deslindaremos convergencias y divergencias.

\section{TGSS: comunicación como sintesis de tres selecciones}

En trabajos previos analizamos el concepto de comunicación de Luhmann, retomamos aquí sus puntos salientes (Pignuoli-Ocampo, 2013a). Según el autor, la comunicación es una síntesis de tres selecciones: InformaciónMitteilung-Verstehen (Luhmann, 1984: 203). Es una operación compleja, cuya unidad emergente se ordena así: una selección de "Información" (el tema, aquello sobre lo que se quiere expresar) es inicialmente procesada por un emisor (alter), quien selecciona un hacer en el mundo (Mitteilung) -una gesticulación, una locución oral, escrita, telecomunicativamente difundida o codificada simbólicamente - para que sea observado por un determinado receptor (ego) hacia quien tal "actuación” es dirigida (Luhmann, 1984: 195-6).

Sin embargo, dado que la comunicación es mutualista, no puede reducirse a la unilateralidad de una emisión, pues requiere que ego "actúe la comprensión" (Verstehen) y distinga entre ese hacer del emisor en el mundo dirigido hacia él (Mitteilung) y su decodificación de la "información”. La decodificación no es pasiva, sino que produce información, y la reintroduce en la unidad de la comunicación. La información de la comunicación no se reduce así al mensaje emitido. Hay comunicación si, y sólo si, ego logra "actuar la comprensión”. Leemos en Luhmann (1984: 203): "Si se entiende la comunicación como síntesis de tres selecciones, como unidad de información, Mitteilung y Verstehen, entonces la comunicación se realiza cuando y hasta donde se genera Verstehen. Todo lo demás sucede "fuera" de la unidad elemental y la presupone".

Al cerrarse sintéticamente, una comunicación sólo podrá ser aceptada o rechazada con una nueva comunicación que enlace con la comunicación anterior. La recursividad de estos enlaces entre comunicaciones genera un límite de sentido que diferencia al sistema social. Por esta razón Luhmann considera la comunicación como la unidad de análisis de la sociología. 
Desde el punto de vista del mutualismo, la TGSS entiende lo social como un fenómeno emergente gracias a, y no contra, la irreductibilidad a los participantes, es decir, gracias a, y no contra, la individualidad de los individuos. Lo social emerge como un orden diferenciado, cuyas propiedades son irreductibles a sus precursores, a los cuales, sin embargo, no deja de presuponer. A su vez, en cuanto diádico, el mutualismo no es ajeno a la interacción, pero reniega de las versiones inespecíficas de este principio, y procura orientarse al problema de la unidad, la diferencia y la alteridad.

\section{TAC: acción comunicativa como éxito ilocutivo}

Habermas (1981-I: 128) ${ }^{6}$ define la acción comunicativa como interacción simbólicamente mediada entre, al menos, dos sujetos capaces de lenguaje y acción que se reconocen intersubjetivamente y que, por medios verbales y/o extraverbales, entablan una relación interpersonal con el propósito de entenderse sobre algo en el mundo y coordinar sus planes de acción. La acción comunicativa supone entonces actos de habla ejecutados por un sujeto en vistas de ser, primero, comprendidos (verstand), y, luego, aceptados y acordados (annehmen) por parte de otro sujeto en situación de habla. La doble estructura de los actos de habla ordena la interacción simbólicamente mediada: el componente locutivo es articulado mediante oraciones gramaticalmente comprensibles referidas a estados de cosas, y el componente ilocutivo es articulado con pretensiones de validez propuestas mediante componentes ilocutivos, éste entabla y regula la relación interpersonal.

Habermas (2002: 117) denomina éxito ilocutivo a la efectiva concreción (einlösen) de un entendimiento entre los actores y la acción comunicativa se alcanza en la medida en que el éxito ilocutivo es logrado, es decir, cuando los actores aceptan las pretensiones de validez propuestas recíprocamente y coordinan sus planes de acción a través del mecanismo del entendimiento lingüístico. Por esta razón, Habermas (1981-I: 151 y 370$)^{7}$ considera que la

6 A diferencia de otros "giros lingüísticos", Habermas no concibe la acción como epifenómeno de las estructuras del lenguaje, sino como una performación interactiva posibilitada por éstas.

7 Alexander (1991: 64ss) criticó a Habermas por confundir comunicación con acuerdo (agreement). Habermas (1991: 238) se defendió señalando, primero, que la TAC, en línea con la pragmática formal distingue, por un lado, Verständigung de Annebmen, distinción que concierne al proceso de alcanzar un acuerdo, y por otro, entre Verstehen y Akzeptabilität, distinción que concierne a la interconexión interna de la comprensión; podríamos agregar que la primera distinción, que ya rebate la crítica de Alexander, es propia del planteo 
consecución del éxito ilocutivo constituye el mecanismo original de coordinación de las acciones sociales, porque es el modo básico en que "ego puede 'conectar' (anschließen) sus acciones con las de alter".

El rechazo de las pretensiones de validez no acaba con la acción comunicativa, sino que abre en ella un ámbito virtual donde las pretensiones de validez son tematizadas con el horizonte de ser justificadas argumentativamente y aceptadas a partir de la racionalización de los motivos y las normas.

El éxito ilocutivo, por tanto, establece la unidad de análisis de la acción comunicativa, primero, por cuanto identifica el mecanismo original del entendimiento lingüístico, segundo, por cuanto permite distinguir entre acción comunicativa débil, cuyo éxito ilocutivo es sostenido por expresiones de voluntad unilaterales y guiado sólo por pretensiones de verdad y veracidad, y acción comunicativa fuerte, cuyo éxito ilocutivo se extiende a razones normativas, que están referidas a orientaciones de valor compartidas, al tiempo que los sujetos se reconocen autonomía y vinculan sus voluntades con independencia de sus preferencias (Habermas, 2002: 117-118); tercero, por cuanto identifica el mecanismo de racionalización de los motivos y las normas propio de la acción comunicativa (fuerte) $;{ }^{8}$ cuarto, por cuanto establece la medida para establecer usos derivados del éxito ilocutivo en otros tipos de acción social, sobre todo el caso de la acción estratégica, cuyo mecanismo de coordinación de la acción no está basado en el entendimiento ni en las razones sino en los intereses, pero que es incapaz de coordinar acciones sin parasitar el éxito elocutivo. Otro tanto sucede con la acción instrumental, que no es en sí una acción social, pero puede ser articulada en plexos de acción comunicativa, y por tanto encuentra en el éxito ilocutivo un mecanismo de coordinación. ${ }^{9}$

\section{Convergencias}

En este elemento hallamos una convergencia sustantiva: la unidad de análisis diádica. Ella rearticula las convergencias en torno al nuevo lugar preparado para lo social, deslindado del accionalismo y del holismo, y el interés por antecedentes disciplinarios de la interacción. Se trata del concepto de constelacondicional en torno a la intersubjetividad del lenguaje, no pertenece a la definición de la unidad de análisis.

8 Habermas (2002: 49) introdujo tardíamente esta distinción a raíz de las críticas de Wellmer (1989) y Lafont (1993) sobre las inconsistencias de su teoría del significado.

9 Habermas (1981-I: 393-4) subrayó que su distinción entre acción comunicativa y acción estratégica no es sólo analítica, sino basada en fundamentos racionales. 
ción que define la unidad social mínima. La elaboración conceptual diádica está presente en la constelación mínima organizada en torno a, al menos, dos alter ego que en virtud de la reciprocidad de sus orientaciones coordinan selecciones.

En el concepto de unidad de análisis esa constelación es definida como un, y sólo un, acontecimiento cualificado como social, que supone pero no se reduce a ninguno de los alter ego constelados (no es monádica), cuya unidad surge únicamente de esa constelación y de ningún componente o condición exterior a ella (no es triádica). Para Luhmann y Habermas es necesario y suficiente el concurso de, al menos, dos alter ego que forman perspectivas recíprocas y se coordinan en un momento y un lugar determinados; a partir de ello, y sólo de ello, es conformado un acontecimiento novedoso, dotado de una duración y una unidad específica e irreductible, relacionalmente contingente.

Tal concurso o constelación cualifica la unidad como social debido a su composición múltiple diádica, como se observa en los conceptos de comunicación de Luhmann y de acción comunicativa de Habermas, más allá de quién (o qué) califique como alter ego para cada uno. Esta convergencia al nivel de la unidad de análisis posee una importancia mayúscula para nuestra investigación, porque sostiene la convergencia teórica en torno a la propiedad diádica de la cualidad sociológica.

Los programas convergen en la definición diádica de la unidad de análisis y la declaran base única de sus sistemas de categorías. De manera que, a partir de la convergencia en la unidad de análisis diádica, rechazan el monadismo, el triadismo y el combinacionismo. En cuanto a la perspectiva monádica, son retomadas las críticas programáticas al individualismo y la crítica ontológica del elementalismo.

Los autores impugnan el monadismo sociológico debido a que, primero, los elementos sociales son unidades, no elementos simples individuales o micro (rechazo del principio de simplicidad); segundo, la constelación no es un agregado de elementos individuales sino una cualidad novedosa (rechazo del principio de aditividad); tercero, las propiedades diádicas no son predicados relativos a propiedades individuales, tales predicados incurren en falacia de nivel equivocado (rechazo del principio de reducción); y cuarto, la constelación diádica no es causada por acciones individuales (rechazo del epifenomenalismo ascendente).

En cuanto a la perspectiva triádica, son retomadas las críticas programáticas al holismo y la crítica ontológica del modelo todo/parte. Ambos autores impugnan el triadismo, o colectivismo sociológico, debido a que, primero, 
la unidad diádica de la constelación no deriva ni guarda correspondencias con una unidad otra anterior o simultánea (rechazo del principio de transitividad); segundo, las propiedades diádicas no son predicados relativos a propiedades colectivas (rechazo del principio de deducción) y tercero, la constelación no es causada por entidades supraindividuales (rechazo del epifenomenalismo descendente).

En cuanto a la perspectiva combinacionista, son retomadas las críticas programática al dualismo y ontológica al agregacionismo. Luhmann y Habermas impugnan el combinacionismo sociológico, debido a que, primero, no hay una meta-unidad sociológica entre dos acontecimientos simultáneos (micro/ macro, acción/estructura) causalmente independientes (rechazo del principio de paralelismo); segundo, las propiedades diádicas no son predicados relativos a la conjunción de propiedades enlazadas (monádico-triádicos), ya que éstas siguen siendo propiedades individuales (monádicas) y propiedades colectivas (triádicas), su conjunción no elimina las críticas precedentes e incurre en falacia de concreción equivocada (confunde su método con lo que observa), el diadismo tiene unidad y facticidad específicas, cuyo orden no surge ni se explica por combinar niveles (rechazo del principio de conjunción), y tercero, la constelación diádica no es efecto de una combinación de causas de otros niveles (rechazo del principio multicausal).

\section{Divergencias}

En este elemento hallamos una divergencia significativa: la segmentación de las unidades de análisis diádicas. Ella radica en la segmentación analítica que cada uno de los autores introdujo en la constelación diádica para distinguir e indicar sus unidades de análisis. Su punto de origen son los criterios analíticos provistos por las perspectivas adoptadas. Éstos, al ser aplicados a la constelación diádica, (la) segmentan (en) unidades de análisis distintas, y explican por qué, aunque conceptualmente diádicas, las unidades de análisis divergen: Luhmann tomó como referencia la unidad de análisis de la perspectiva sistémica y segmentó unidades diádicas operativas, mientras Habermas hizo lo propio con la unidad de análisis de la perspectiva orientada al entendimiento y segmentó unidades diádicas de acuerdo. Esta segmentación divergente es relevante porque identifica el punto en que las perspectivas generan divergencias entre concepciones convergentes.

Esta divergencia general explica las divergencias particulares entre Luhmann y Habermas al respecto de la comunicación y por ello merece un párrafo aparte. Ambos encuadran el diadismo en la comunicación y sin 
embargo la unidad de análisis de la TAC y la TGSS difiere. Ello no se explica por un presunto accionalismo del primero ni un presunto holismo del segundo, como sugieren las recepciones recíprocas, sino por la distinta segmentación de la constelación diádica. Es decir, la divergencia no surge de la composición del argumento, pues en ello los autores no divergen, para los dos hay constelación diádica, sino que en la segmentación y la base de ello está la distribución de dos componentes fundamentales, por un lado, el componente Verstehen ("comprensión") o Verständlichkeit ("comprensibilidad", "inteligibilidad"), y por otro, el componente annehmen/Annabme o Akzeptabel ("aceptar", "aceptación").

Tanto Luhmann (1984: 203) como Habermas (1984: 209), lo reiteramos, asumen conceptos sobre una base diádica y los diferencian entre sí: el primero ("comprensión") implica para ambos la asunción de la orientación hacia ego por parte de alter, el segundo ("aceptación”) implica para ambos el no rechazo de alter de una propuesta de ego. Sin embargo, los distribuyen divergentemente en relación con sus unidades de análisis respectivas: Luhmann incluye el concepto de Verstehen en la unidad de análisis, lo considera la tercera selección de la comunicación, cuya unidad se cierra con él, mientras excluye de ella el concepto de Annabme, al que considera una cuarta selección cuyo valor es procesal.

Habermas, en cambio, incluye el concepto de Annabme en la unidad, lo considera la concreción de la coordinación de la acción, cuya unidad se cierra con él, mientras aloja el concepto de Verständlichkeit en el alter ego y en la construcción de la oración, sólo ligado con la unidad del entendimiento de modo condicional y subordinado mediante la teoría del significado ("comprendemos un acto de habla cuando sabemos qué lo hace aceptable").

La distinta distribución de estos componentes explica la distinta segmentación de las unidades de análisis: para la "síntesis de tres selecciones" de la TGSS basta la comprensión, mientras que la "acción comunicativa" de la TAC exige aceptación. A partir de ello divergen los métodos aplicados a las pretensiones. Luhmann plantea un concepto universal de unidad de análisis, que abarca todo lo relativo al campo, y Habermas un concepto general, común a los particulares, que enfatiza la originalidad de la acción comunicativa, de la que se derivan las otras formas de acción social, en esa originalidad pone en juego las pretensiones y la pertinencia de la racionalidad para el objeto y su campo.

Esta divergencia en la segmentación de las unidades de análisis origina una larga cadena de divergencias entre la TAC y la TGSS. No obstante, aquí nos interesa señalar que la divergencia entre pretensiones programáticas 
tiene un efecto multiplicador sobre ella. Schluchter (2008: 49) sintetizó con acierto la cuestión: "Luhmann exige demasiado poco del potencial de racionalidad de la comunicación, Habermas exige demasiado". Esto se debe a que la pretensión de universalidad de Habermas sobre la racionalidad entra en escena gracias a una segmentación que incluye la cuestión de la racionalidad en la unidad de lo social por la vía de la aceptación o fundamentación de las pretensiones de validez, con lo que estableció un umbral relativamente alto para la formación de unidades sociales diádicas.

La pretensión de universalidad de Luhmann, en cambio, lo llevó a subsumir la racionalidad de los motivos como un caso posible de síntesis de tres selecciones, con ello estableció un umbral relativamente bajo para la emergencia de unidades sociales diádicas. En otros términos, al independizar la unidad de análisis respecto de la aceptación, la TGSS ingresa al campo del objeto antes que la TAC, con lo cual tiene un acceso inicial más amplio a él.

Pero la TAC, al declararla dependiente, introduce el problema del orden social como dimensión normativa al nivel de las unidades de análisis, problema que la TGSS desdobla: distribuye el problema del orden social al nivel del planteo condicional, la aceptación al nivel procesal y la normatividad al nivel de la modulación de estructuras. Ninguno queda al nivel de la unidad de análisis.

\section{Conclusiones: Diadismo marcado y multinivelado}

A lo largo del trabajo cotejamos las definiciones comunicativas de "lo social" de Luhmann y Habermas. En todos los ejes de análisis hallamos convergencias y divergencias, con una significativa diferencia en número y calidad a favor de las primeras. Si bien en el planteo condicional se equiparan, ponderamos las convergencias porque rebaten las recepciones recíprocas y los estándares interpretativos. Con este énfasis no negamos las divergencias, más bien las ponderamos. En la Tabla 2 ordenamos los resultados.

A juzgar por los resultados, concluimos que las unidades de análisis comunicativas de la TGSS y la TAC guardan muy importantes puntos de similitud en criterios de socialidad, planteos condicionales y unidad de análisis, y un punto de contraste significativo en torno a la segmentación de las unidades diádicas. Las similitudes indican convergencias entre las definiciones de "lo social", abocadas a elaborar conceptualmente un esquema sociológico fuertemente diádico en todos los niveles de la definición; mientras que el punto nodal de las divergencias son las decisiones analíticas relativas a esa base. 
Esto es particularmente evidente en la segmentación de unidades de análisis sobre la constelación alter ego / alter ego. Subrayamos que las divergencias no surgen de la base sociológica, pues ambos programas asumen una base diádica y critican al monadismo y al triadismo.

Vistas sistemáticamente, las unidades de análisis tienen un elemento común y uno contrapuesto. En cuanto al primero, en los tres ejes se observa una elaboración orientada al diadismo; ella establece una cualidad sociológica con marcada contraposición al monadismo, al triadismo y al combinacionismo, este último considerado una agregación de los dos primeros. En cuanto al elemento contrapuesto, la segmentación de la unidad de análisis ubica las divergencias al nivel de la unidad mínima analítica.

De estos elementos se sigue que los programas elaboraron en paralelo, pero de modo convergente, un mismo punto de partida: criticar las "tradiciones sociológicas" y definir "lo social” de un modo marcadamente diádico. Esto es, a su vez, congruente con una base ontológicamente débil y fácticamente fuerte. En esta conclusión convergente se articulan las críticas al monadismo, triadismo y combinacionismo con la opción por un diadismo muy marcado al nivel de los criterios de socialidad, los planteos condicionales y las unidades de análisis. ${ }^{10}$

Este es un punto de partida común puesto que tolera una diversidad de planteos condicionales y segmentaciones de las unidades de análisis, todos igualmente diádicos, hasta el punto que cabe considerarlos equivalentes funcionales diádicos. Esta articulación sistemática evita una trampa de las recepciones recíprocas, que consiste en acusar, o bien de "accionalista" a cualquier programa que emplee apenas el término "acción”, hecho que implicaría para Luhmann una concesión al monadismo, o bien de "holista" a cualquier programa que emplee el término "sistema", hecho que implicaría para Habermas una concesión al triadismo. Nuestra conclusión sistemática es muy diferente: ambos programas rechazan de manera convergente las tradiciones sociológicas y aceptan una definición radicalmente diádica de "lo social"; lo que resulta congruente con nuestra hipótesis inicial.

10 Estos resultados pueden triangularse con los alcanzados en el cotejo de Luhmann y Latour, donde también hallamos una fuerte convergencia en torno al diadismo en las bases sociológicas. Así los planteos se ampliarían recíprocamente y confluirían con las convergencias en torno al "cambio de paradigma”. 


\section{Referencias}

Albert, Gert et al. (2010), Dimensionen und Konzeptionen von Sozialität, Alemania: VS Verlag.

Alexander, Jeffrey (1991), "Habermas and Critical Theory: Beyond the Marxian Dilemma?", en Honneth, A. y Joas, H., Communicative Action, Estados Unidos: MIT Press.

Austin, John (1982), ¿Cómo hacer cosas con palabras?, España: Paidós.

Bedorf, Thomas et al. (2010), Theorien des Dritten. Innovationen in Soziologie und Sozialphilosophie, Alemania: Wilhelm Fink.

Bolz, Norbert (2012), Ratten im Laberynth, Alemania: Wihelm Fink.

Bonacker, Thorsten (2002), "Die Rekonstruktion der soziologischen Vernunft", en Der soziologische Blick, Alemania: Leske und Budrich.

Colomy, Paul (1991), "Metatheorizing in a Postpositivist Frame", en Sociological Perspectives, vol. 34, núm. 3, Estados Unidos: University of California Press.

Chernilo, Daniel (2002), "The Theorizations of Social Co-ordinations in Differentiated Societies”, en The British Journal of Sociology, vol. 53, núm. 3, Inglaterra: London School of Economics.

Habermas, Jürgen (1981), Theorie des kommunikativen Handelns, Alemania: Suhrkamp.

Habermas, Jürgen (1984), Vorstudien und Ergänzungen zur Theorie des kommunikativen Handelns, Alemania: Suhrkamp.

Habermas, Jürgen (1991), "A Reply”, en Honneth, A. y Joas, H., Communicative Action, Estados Unidos: MIT Press.

Habermas, Jürgen (2002), Verdad y justificación. Ensayos filosóficos, España: Trotta.

Heintz, Bettina (2004), "Emergenz und Reduktion”, en Kölner Zeitschrift für Soziologie und Sozialpsychologie, vol. 56, núm. 1, Alemania: Universität Köln.

Lafont, Cristina (1993), La razón como lenguaje, España: Visor.

Lindemann, Gesa (1999), "Doppelte Kontingenz und reflexive Anthropologie”, en Zeitschrift für Soziologie, vol. 28, núm. 3, Alemania: Universität Bielefeld.

Lindemann, Gesa (2006), "Die Emergenzfunktion und die konstitutive Funktion des Dritten”, en Zeitschrift für Soziologie, vol. 35, núm. 2, Alemania: Universität Bielefeld.

Luhmann, Niklas (1984), Soziale Systeme, Alemania: Suhrkamp.

Luhmann, Niklas (1990), "Autopoiesis of Social Systems", en Luhmann, N., Essays on SelfReference, Estados Unidos: Columbia University Press.

Maciejewski, Franz [comp.] (1974), Theorie der Gesellschaft oder Sozialtechnologie. Beiträge zur Habermas-Lubmann-Diskussion. Supplement 2, Alemania: Suhrkamp.

Mascareño, Aldo (2008), "Acción, estructura y emergencia en la teoría sociológica”, en Revista de Sociologia, núm. 22, Chile: Universidad de Chile.

Mascareño, Aldo (2009), "Medios Simbólicamente Generalizados y el Problema de la Emergencia”, en Cinta de Moebio, núm. 36, Chile: Universidad de Chile.

Paladino, Federico y Pignuoli-Ocampo, Sergio (2014), "Convergencias y divergencias entre los conceptos de interpenetración de Luhmann y de intersubjetividad de Habermas", Ponencia presentada en VIII Jornadas de Sociología de la UNLP, Argentina.

Pignuoli-Ocampo, Sergio (2013a), "El modelo sintético de comunicación de Niklas Luhmann”, en Cinta de Moebio, núm. 47, Chile: Universidad de Chile.

Pignuoli-Ocampo, Sergio (2013b), "Doble contingencia y orden social desde la teoría de sistemas de Niklas Luhmann”, en Sociológica, núm. 78, México: Universidad Autónoma Metropolitana. 
Pignuoli-Ocampo, Sergio (2015a), "Las fenomenologías post-husserlianas de Alfred Schutz y Niklas Luhmann: soluciones para el problema de la intersubjetividad", en Revista Internacional de Sociología, núm. 73, vol. 3, España: Instituto de Estudios Sociales Avanzados.

Pignuoli-Ocampo, Sergio (2015b), “La disputa por el 'cambio de paradigma' en Luhmann, Latour y Habermas”, en Estudios sociológicos, núm. 99, México: El Colegio de México.

Pignuoli-Ocampo, Sergio (2016a), "Diadismo en los fundamentos sociológicos de Luhmann y Latour”, en Revista Española de Investigaciones Sociológicas, núm. 155, España: Centro de Investigaciones Sociológicas.

Pignuoli-Ocampo, Sergio (2016b), “Aportes de las teorías sociológicas a la discusión de la ontología. Los casos de Luhmann, Habermas y Latour”, en Revista de Filosofía, vol. 41, núm. 1, España: Universidad Complutense.

Pignuoli-Ocampo, S. (2017), "La perspectiva del programa de investigación multinivelado como metodología de teoría sistemática”, en Revista Mexicana de Sociología, vol. 79, núm. 2, México: Universidad Nacional Autónoma de México.

Pignuoli-Ocampo, Sergio y Zitello, Matías (2008), "Estatutos sociológicos del concepto de comunicación en el campo de la cultura”, en Latitude, vol. 2, núm. 1, Brasil: Universidade Federal do Alagoas.

Rasch, William (2000), Niklas Luhmann's Modernity, Estados Unidos: SUP.

Ritzer, George (2001), Explorations in Social Theory, Inglaterra: Sage.

Schluchter, Wolfgang (2008), Acción, orden y cultura, Argentina: Prometeo.

Söllner, Alfons (1982), "Jürgen Habermas und die kritische Theorie des gegenwärtigen Rechtsstaates”, en Leviathan, vol. 10, núm. 1, Alemania: Nomos Verlagsgesellschaft $\mathrm{mbH}$.

Strydom, Piet (1999), "Triple contingency: The theoretical problem of the public in communication Societies", en Philosophy \& Social Criticism, vol. 25, núm. 2, Inglaterra: Sage.

Vanderstraeten, Raf(2002), "Parsons, Luhmann and the Theorem of Double Contingency", en Journal of Classical Sociology, vol. 2, núm. 1, Inglaterra: Sage.

Wellmer, Albrecht (1989), “Was ist eine pragmatische Bedeutungstheorie?”, en Honneth, A. et al. [coords.], Zwischenbetrachtungen. Im Prozeß der Aufklärung, Alemania: Suhrkamp. 
Anexo

\section{Tabla 1}

Esquema de equivalencias entre las definiciones de "lo social"

\begin{tabular}{lll}
\hline & Luhmann & Habermas \\
\hline Criterio de socialidad & Mutualismo & $\begin{array}{l}\text { Reconocimiento } \\
\text { intersubjetivo }\end{array}$ \\
\hline Planteo condicional & Doble contingencia & $\begin{array}{l}\text { Fuerza ilocutiva } \\
\text { del lenguaje }\end{array}$ \\
\hline Unidad de análisis & 'Comunicación' & $\begin{array}{l}\text { Acción comunicativa } \\
\text { síntesis de tres selecciones }\end{array}$ \\
\hline
\end{tabular}

Fuente: Elaboración propia.

Tabla 2

Convergencias y divergencias entre definiciones de "lo social"

\begin{tabular}{lll}
\hline & Convergencias & Divergencias \\
\hline Criterio de socialidad & $\begin{array}{l}\text { Base interaccional, situacio- } \\
\text { nal y recíproca } \\
\text { Centralidad de alteridad }\end{array}$ & Acentos Interaccionistas \\
\hline Planteo condicional & $\begin{array}{l}\text { Doble uso del criterio de } \\
\text { socialidad }\end{array}$ & Modulación del planteo \\
& $\begin{array}{l}\text { Inclusión de tercer término } \\
\text { condicional }\end{array}$ & \\
\hline Unidad de análisis & $\begin{array}{l}\text { Unidad única de análisis } \\
\text { diádica }\end{array}$ & $\begin{array}{l}\text { Segmentación de la unidad de } \\
\text { análisis diádica }\end{array}$ \\
\hline
\end{tabular}

Fuente: Elaboración propia. 
Sergio Pignuoli Ocampo. Doctor en Ciencias Sociales (CONICETUBA-IIGG), Investigador Asistente del CONICET y docente de la cátedra "Niklas Luhmann y la sociología de la modernidad" (FCS-UBA). Líneas de investigación: teoría sociológica, teoría de sistemas sociales y comunicación. Publicaciones recientes: "Diadismo en los fundamentos sociológicos de Luhmann y Latour", en Revista Española de Investigaciones Sociológicas, núm. 155, Madrid, España: Centro de Investigaciones Sociológicas (2016); "Las fenomenologías post-husserlianas de Alfred Schutz y Niklas Luhmann”, en Revista Internacional de Sociología, vol. 73, núm. 3, La Rioja, España: Instituto de Estudios Sociales Avanzados (2015); "El programa sociológico de Niklas Luhmann y su contexto" en Revista Mexicana de Sociologia, vol.77, núm. 2, México D.F., Universidad Nacional Autónoma de México (2015).

Recepción: 27 de octubre de 2014.

Aprobación: 12 de octubre de 2016. 